\title{
55-year old woman with erysipelas
}

\author{
Katarzyna Plagens-Rotman ${ }^{1}$, Renata Przybylska², Katarzyna Gerke³ ${ }^{3}$ Zygmunt Adamski ${ }^{3}$, Magdalena Czarnecka-Operacz $^{3}$
}

${ }^{1}$ Institute of Health Sciences, Hipolit Cegielski State College of Higher Education, Gniezno, Poland 2Department of Neurological Nursing, Poznan University of Medical Sciences, Poznan, Poland ${ }^{3}$ Department of Dermatology, Poznan University of Medical Sciences, Poznan, Poland

Adv Dermatol Allergol 2020; XXXVII (4): 613-616 DOI: https://doi.org/10.5114/ada.2020.98225

Erysipelas belongs to the inflammatory erythema of acute course spreading with lymphatic vessels, in which the pathogen (group A $\beta$-haemolytic streptococcus) penetrates the human system through the extrinsic (skindamaging) or intrinsic pathway, in particular from nasal mucosa and oral cavity, which in turn leads to infection of the skin and subcutaneous tissue. The frequency of occurrence of erysipelas in European countries is estimated at 9-24 per 10,000 inhabitants [1, 2]. The peak of illness is between 60 and 80 years of age, with women suffering more often than men. In some cases, a predisposition to the disease among newborns and young children is also observed [3].

In most of the cases, the etiological factor is group A $\beta$-haemolytic streptococcus (Streptococcus pyogenes gr. A, GAS) observed in $58-67 \%$ of cases $[4,5]$, less frequently $\mathrm{G}$ group streptococci (14-25\%) or streptococci of group B (3-9\%). It must be emphasized that B group streptococci play a significant role after gynaecological procedures or in patients treated with radiotherapy at gynaecologic oncology wards [6].

The onset of the disease is sudden. There is a high fever reaching $40^{\circ} \mathrm{C}$ accompanied by chills. There is an acute inflammation of the skin and subcutaneous tissue with erythema and oedema present. The focus of the disease is characterized by a smooth surface limited from the environment by a steep, clearly marked heaped-up edge. In addition, streaky protrusions are often caused by the spread of infection via the lymphatic vessels. The complication of the accumulation of exudative fluid in the papillary layer is the separation of the epidermis and the formation of blisters (erysipelas bullosum). In addition, the severe course of erysipelas may lead to necrosis and gangrene (erysipelas gangraenosum) [7, 8].

Diagnosis of erysipelas is based on the characteristic clinical picture. Laboratory tests show an elevated erythrocyte sedimentation rate test and leucocytosis with an increased percentage of polymorphonuclear neutrophils.
Antibiotics of choice in the treatment of erysipelas are semi-synthetic penicillins, however, in the case of intolerance or allergies to this group of drugs, the recommended treatment is erythromycin.

The aim of the report was to present a case of erysipelas in a 55-year-old woman treated at the Department of Dermatology, Poznan University of Medical Sciences.

A 55-year-old woman was admitted to hospital as a matter of urgency at the Department of Dermatology for the purpose of diagnosis and treatment with the diagnosis of facial erysipelas. Medical history from several days before was taken. Punctual pain of the nose and left submandibular lymph node was also noted.

The patient was consulted otolaryngologically, which showed no change. Afterwards, increasing swelling and redness of the skin of the nose and left half of the face occurred with a feeling of pulsation accompanied by chills (fever of $38^{\circ} \mathrm{C}$ ). Doxycycline, Co-trimoxazole, Mupirocin ointment were administered but without improvement of the dermatological condition. The family history for skin diseases was negative. The patient was not treated internally either.

The patient's history included chronic sinusitis (paranasal sinuses) and Kartagener's syndrome. In addition, in April 2015, the patient was hospitalized at the department of internal diseases for the purpose of observation in the direction of systemic lupus, but it proved negative.

During the hospital stay the patient was consulted: - dentally: In the clinical examination and after radiological analysis, there are potential outbreaks in the oral cavity. Teeth 27 and 28 require the extension of radiological diagnosis and possibly endodontic treatment or extraction; apart from that, changes of the left maxillary sinus were observed.

- laryngologically: recurrent nasal polyps in the interview - 3 functional endoscopic sinus surgeries (FESS) (last in 2014); ears - otoscopic examination - not changes found; nose - patent, small polyp change emerging

Address for correspondence: Katarzyna Plagens-Rotman, Institute of Health Sciences, Hipolit Cegielski State College of Higher Education, 38 Ks. Kard. S. Wyszyńskiego St, 62-200 Gniezno, Poland, phone: +61 4242942, e-mail: plagens.rotman@gmail.com Received: 20.11.2018, accepted: 26.04.2019. 
from the left side ethmoid bone, not disturbing nasal patency; oral cavity and throat - after tonsillectomy, smooth. The above-mentioned changes in the sinuses X-ray may correspond to chronic changes of the paranasal sinuses; it was recommended to have computed tomography (CT) of sinuses in the bone window to accurately assess the paranasal sinuses.

The following imaging tests were performed:

- chest X-ray: lung fields with no visible focal lesions; cardiac and right-aortic silhouette, not enlarged cardiac silhouette, aorta in norm; unveiled, vascular pulmonary hila; air bubble under the right dome of the diaphragm - which may suggest visceral inversion.

- abdominal ultrasound: visceral total inversion. Liver of normal echogenicity, not enlarged, with no visible focal lesions; non-dilated bile ducts; gallbladder, thin-walled without any deposits. In the shank of the gallbladder, a parietal soft tissue echo of $4 \mathrm{~mm}$ height was seen - polyps ultrasound image - an ultrasound scan control for 6-12 months; not enlarged pancreas with normal parenchyma echogenicity, non-dilated pancreatic duct; kidneys of normal structure and shape without stagnation and echoes of deposits; hyperechogenic focal lesion of $4 \mathrm{~mm}$ diameter in the middle part of the parenchymal layer of the right kidney - ultrasound image may correspond to angiomyolipoma (AML) - ultrasound scan control recommended. Spleen homogeneous, not enlarged. Urinary bladder not full.

- sinuses CT: state after multiple FESS - with a wide opening of both maxillary sinuses to the nasal cavity and bilateral turbinectomy. Parietal polypo-mucosal lesions up to $14 \mathrm{~mm}$ thick present in the maxillary sinuses. Parietal thickening of the mucosa also visible within the ethmoid area and in hypoplastic ostia of the frontal sinuses. Minimal segmental thickening of the sphenoidal sinuses mucosa. Patent nasal cavity and posterior nostrils. Ostiomeatal complex unobstructed. Swelling of the mucous membrane of inferior nasal concha.

Laboratory tests performed during hospitalization show elevated fasting glucose $(115 \mathrm{mg} / \mathrm{dl})$ and C-reactive protein (54.5 mg/dl).

The patient was treated with the following medications: Amoxicillin $2 \times 1,2 \mathrm{~g}$ i.v. for 10 days, Metronidazole $3 \times 500 \mathrm{mg}$ i.v., probiotic once a day, Hydrocortisone $2 \times 200 \mathrm{mg}-1 \times 200 \mathrm{mg}-100 \mathrm{mg}$, Potassium chloride $1 \times 1$, Pantoprazole; Proton pump inhibitors (PPIs) $20 \mathrm{mg}$ $1 \times 1$, Enoxaparin sodium $0.4 \mathrm{ml}$ subcutaneously and boric acid wraps on the changed skin and Mupirocin.

The patient was discharged home in good general condition with the following recommendations:

- post-hospital control in the Dermatological Outpatient Clinic after 1 month;

- further care of the general practitioner (GP): control of blood pressure and blood glucose, blood morphology, fasting glucose, electrolytes;

- basic diet;

- physical exertion suitable for patient's age.
The most common etiological agent causing erysipelas is Gram-positive granulomas - $\beta$ streptococci-haemolytic group A (Streptococcus pyogenes) belonging to more than 50 species of the Streptococcus type. During the infection, the pro-inflammatory cytokine is released (HMGB1 protein) causing the activation of macrophages, NK and NKT cells and contributing to the stimulation of T lymphocytes [9]. Transmission of the microorganism can occur via droplets, through direct contact with mucus secretion from the nasopharynx of the sick person or the carrier, as well as through infected wounds or pathologically altered skin.

The most common infections caused by Streptococcus pyogenes include streptococcal angina, scarlet fever, sinusitis, otitis media, erysipelas, purulent dermatitis and purulent inflammation of subcutaneous tissue, neonatal navel infection, nodular inflammation and lymphatic vessels or toxic Streptococcal like syndrome (TSLS). Meningitis (post-traumatic meningitis), endocarditis, pneumonia or arthritis and osteoarthritis are diagnosed more rarely [10-12].

Staphylococcus aureus, Streptococcus pneumonia or Pseudomonas aeruginosa should be mentioned among the etiological agents in the development of erysipelas. In case of facial changes, Haemophilus influenzae may play a particularly important role [13]. In addition, the general condition of the patient, the presence of comorbidities, such as alcohol addiction, obesity, diabetes, circulatory insufficiency, kidney failure, long-term antibiotic therapy, treatment with cytostatics, glucocorticoids, immunosuppression, radical mastectomy or advanced age of the patient are all important risk factors for developing erysipelas [1, 14, 15].

Dermatological changes include the skin and subcutaneous tissue in the form of swelling, redness and a clear, heapedup edge limiting it from a healthy tissue. Inflammation is characterized by irregular shape, significant consistency and pain. In addition, high body temperature, chills, tachycardia, increased sweating, headaches, muscle and joint pain belong to the characteristic symptoms which are the consequence of inflammation caused by infection. In the case of the face, the erythematous change usually starts on the bridge of the nose, spreading symmetrically to the cheeks, often with massive eyelid oedema. Very rarely the chin area and lower lip are affected $[6,16,17]$.

Diagnosis of erysipelas is based on the accompanying acute inflammation, clearly separated from the surrounding healthy tissues, sudden and rapid course and a sharp increase in body temperature with chills. In order to make a correct diagnosis it is necessary in case of cutaneous lesions located on the limbs to distinguish the erysipelas from thrombophlebitis with percutaneous inflammation of the skin (peri-et thrombophlebitis) characterized by induration and pain in the vein, less restricted inflammation and to distinguish from erythema 
nodosum differing from the environment with general symptoms with a slow course. In the case of the location of dermatological lesions within the face, the erysipelas is differentiated with organ erythematosus (SLE), which, when exposed to sun may resemble erysipelas, with herpes zoster (zoster) characterized by pain and recurrent erysipelas (erysipelas carcinomatosum). In addition, it is recommended to consider a microbiological examination of swabs taken from skin damage or cracks to determine the factor causing the infection [18-20].

Histopathological examination of dermatological lesions in the course of erysipelas is performed rarely, most often in the case of resistance to antibiotic therapy with pronounced skin oedema, inflammatory infiltrates composed of leukocytes and lymphocytes with the occurrence of acidophilic leukocytes. In the initial stage of the disease, inflammation affects the skin, sometimes the subcutaneous tissue, which results in dilatation of blood vessels and lymphatic vessels $[21,22]$.

In the treatment of rose, antibiotics of choice are natural penicillin - phenoxymethylpenicillin (penicillin $\mathrm{V}$ ) in a dose of 1.2-1.5 million. IU administered by the oral route for 10-14 days with dermatological control after 2-3 days from the beginning of treatment. In case of allergy to $\beta$-lactam antibiotics, it is recommended to take oral erythromycin at a dose of $2 \mathrm{~g} /$ day or intravenously at a dose of $300 \mathrm{mg}$ every $8 \mathrm{~h}$. Considering the presence of factors predisposing to the therapy, it is recommended to perform it in a hospital setting, implementing penicillin $\mathrm{G}$ treatment at a dose of 10-20 million units daily administered in intravenous infusions 2-6 times a day or with procaine penicillin intramuscularly at a dose of 2.4 million units 2 times a day for a period of 10-14 days. [23-25]. In addition, topical treatment involves the use of $2 \%$ ichthyol or $3 \%$ boric acid wraps, while in the presence of blisters, erosions or ulcers, disinfectants such as Polseptol, Octenisept and others are used.

Nursing care of the patient with erysipelas:

- in the case of lower extremity erysipelas, clothes which cause pressure during the acute phase should be avoided, however they should be reapplied as soon as the condition of the affected body part permits;

- the use of NSAIDs, e.g. ibuprofen, during the acute phase of the disease should be avoided as they may cause necrotizing fasciitis;

- return to work should be dependent on the patient's type of job;

- prevention of injuries;

- treatment of ulcerations, mycosis and venous insufficiency;

- weight reduction;

- glycaemic control.

\section{Conflict of interest}

The authors declare no conflict of interest.

\section{References}

1. Inghammar M, Rasmussen M, Linder A. Recurrent erysipelas-risk factors and clinical presentation. BMC Infect Dis 2014; 14: 270.

2. Bartholomeeusen S, Vandenbroucke J, Truyers C, Buntinx F. Epidemiology and comorbidity of erysipelas in primary care. Dermatology 2007; 215: 118-22.

3. Neri I, Montanari F, Baraldi C, et al. Erysipelas as a superinfection of an oral lymphangioma. J Pediatr 2014; 165: 205.

4. Bläckberg A, Trell K, Rasmussen M. Erysipelas, a large retrospective study of aetiology and clinical presentation. BMC Infect Dis 2015; 15: 402.

5. Stevens DL, Bisno AL, Chambers HF, et al. Practice guidelines for the diagnosis and management of skin and soft tissue infections: 2014 update by the Infectious Diseases Society of America. Clin Infect Dis 2014; 59: e10-52.

6. Sunderkötter C, Becker K. Frequent bacterial skin and soft tissue infections: diagnostic signs and treatment. J Dtsch Dermatol Ges 2015; 13: 501-24.

7. Pereira de Godoy JM, Galacini Massari P, Yoshino Rosinha M, et al. Epidemiological data and comorbidities of 428 patients hospitalized with erysipelas. Angiology 2010; 61: 492-4.

8. Solomon M, Barzilai A, Elphasy H, et al. Corticosteroid therapy in combination with antibiotics for erysipelas. Isr Med Assoc J 2018; 20: 137-40.

9. Johansson L, Snall J, Sendi P, et al. HMGB1 in severe soft tissue infections caused by Streptococcus pyogenes. Front Cell Infect Microbiol 2014; 4: 4.

10. Shaikh N, Leonard E, Martin JM. Prevalence of streptococcal pharyngitis and streptococcal carriage in children: a metaanalysis. Pediatrics 2010; 126: e557-64.

11. Little P, Stuart B, Hobbs FD, et al. Predictors of suppurative complications for acute sore throat in primary care: prospective clinical cohort study. BMJ 2013; 347: f6867.

12. Matthys J. There are still problems in identifying who will develop complications of sore throat in primary care. BMJ 2014; 348: g299.

13. Atzori L, Manunza F, Pau M. New trends in cellulitis. EMJ Dermatol 2013; 1: 64-76.

14. Krasagakis K, Samonis G, Valachis A, et al. Local complications of erysipelas: a study of associated risk factors. Clin Exp Dermatol 2011; 36: 351-4.

15. Lundstedt D, Gustafsson M, Malmström P, et al. Symptoms 10-17 years after breast cancer radiotherapy data from the randomised SWEBCG91 - RT trial. Radiother Oncol 2010; 97 : 281-7.

16. Fokina EG, Gerasimov AN. Prediction degree severity of erysipelas: risk index of hemorrhagic form of erysipelas. J Infect Dis Treat 2016; 2: 2.

17. Krasagakis K, Valachis A, Maniatakis P, et al. Analysis of epidemiology, clinical features and management of erysipelas. Int J Dermatol 2010; 49: 1012-7.

18. Crisp JG, Takhar SS, Moran GJ, et al. Inability of polymerase chain reaction, pyrosequencing, and culture of infected and uninfected site skin biopsy specimens to identify the cause of cellulitis. Clin Infect Dis 2015; 61: 1679-87. 
19. Li DG, Xia FD, Khosravi H, et al. Outcomes of early dermatology consultation for inpatients diagnosed with cellulitis. JAMA Dermatol 2018; 154: 537-43.

20. Ko LN, Garza-Mayers AC, St John J, et al. Effect of dermatology consultation on outcomes for patients with presumed cellulitis: a randomized clinical trial. JAMA Dermatol 2018; 154: 529-36.

21. Kihiczak GG, Schwartz RA, Kapila R. Necrotizing fasciitis: a deadly infection. J Eur Acad Dermatol Venereol 2006; 20 : 365-9.

22. Trebing D, Göring HD. Wound healing of chronic leg ulcers under the influence of erysipelas. Eur J Dermatol 2004; 14: 56-7.

23. Kilburn SA, Featherstone P, Higgins B, Brindle R. Interventions for cellulitis and erysipelas. Cochrane Database Syst Rev 2010; 6: CD004299.

24. Thomas KS, Crook AM, Nunn AJ, et al. Penicillin to prevent recurrent leg cellulitis. N Engl J Med 2013; 368: 1695-703.

25. Oh CC, Ko HC, Lee HY, et al. Antibiotic prophylaxis for preventing recurrent cellulitis: a systematic review and metaanalysis. J Infect 2014; 69: 26-34. 